\title{
Mechanical Property Analysis of Two Kinds of Steel Wire Reinforced Composite Pipes
}

\author{
Peng Shang, Anping Xu* , Jingjing Zhao \\ School of Mechanical Engineering, Hebei University of Technology, Tianjin 300130, China \\ *Corresponding authors'Email: xap@hebut.edu.cn
}

\begin{abstract}
Recently the steel wire reinforced composite pipelines are increasingly used in many different fields with researches usually focus on their mechanical properties. A constitutive model of the composite pipe's mechanical properties is presented, which can be used to solve the problems of stress, strain and displacements. After that, 3D CAD models of two kinds of steel wire reinforced composite pipes are built, and then the displacements and stress distribution of the pipes under constant internal and external pressure are analyzed by using FEA software, respectively. The differences between the mechanical properties of the two kinds of composite pipes are elaborated by comparing the displacements of the distribution curve along their corresponding paths. The results show that the braided steel wire reinforced composite pipes have better mechanical properties than the welded ones. That may be due to the crisscross that makes both the clockwise and counter-clockwise steel wires bear the same pressure.
\end{abstract}

Keywords: Steel wire reinforced composite pipe; FEA; mechanical property; composite material; braided steel wire reinforced composite pipe; welded steel wire reinforced composite pipe

\section{Introduction}

Recently the steel wire reinforced composite pipelines are increasingly used in many different fields. The machining process of the composite pipelines is as follows: An inner plastic layer is extruded firstly; then the steel wires are wound around its surface as the reinforced layer; finally they are totally overlaid with a layer of the plastic. There are mainly two kinds of composite pipes: One is the braided steel wire reinforced composite pipe, in which the reinforcement steel wires are woven covering the plastic surface in an constant helix angle with respect to the pipe axis, as shown in Figure 1(a); the other is the welded steel wire reinforced composite pipe, in which the reinforcement steel wires are divided into longitudinal and latitudinal groups that are bonded by spot welding, as shown in Figure 1(b). In both kinds of composite pipes, the steel wires are totally embedded into the plastic, and therefore the two different materials com- bine together perfectly. The composite pipelines have the advantages of both metal and plastic, such as good mechanical properties of metal and good chemical properties of plastic. Therefore the composite pipelines are widely used in areas such as water supply and drainage, heat supply, chemical engineering, petroleum and natural gas transportation, etc.

Nowadays, the researchers usually focus on their mechanical properties. Western Europe and the U.S. lead in the processing technology and performance analysis of the pipelines. The mechanics properties of composite pipe under internal pressure and bending moment were discussed by Xia et al. [1]. The theory used by H. Bakaiyan et al. [2] to analyze the mechanical performances of multi-layered filamentwound composite pipelines under combined internal pressure and thermal loading also can be applied to the steel wire reinforced composite pipelines.

Meanwhile it also attracts much attention in china. 


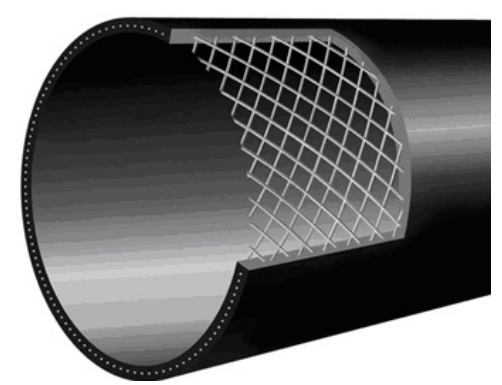

(a)

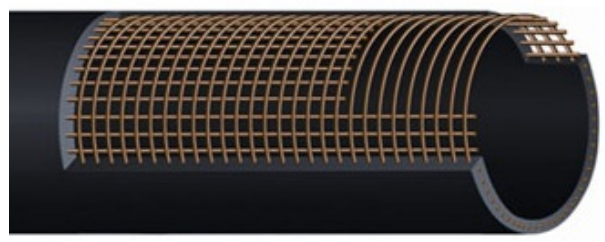

(b)

Figure 1 Steel wire reinforced composite pipelines: (a) Braided steel wire reinforced composite pipe, and (b) welded steel wire reinforced composite pipe

Lots of patents on the steel wire reinforced composite pipes have been authorized. Li [3] reported a kind of heavy-duty multi-layer steel-plastic composite pipe; Gan [4] developed a kind of spiral wound steel wire reinforced plastic composite pipe; Huang [5] invented the all buried continuous winding reinforced plastic composite pipes. In theoretical and experimental research, the static pressure process small equality of static pressure blasting tests under stress distribution and deformation pipelines, analyzed by finite element method [6]. Zheng et al. [7] studied the properties of steel wire reinforced plastic pipe under short-term blasting pressure, strain, buckling critical pressure and the different temperature failure forms. The domestic research and production department of composite pipe paid much attention on production technology, and the government departments have enacted a series of related standards $[8,9]$.

Currently producers and users usually concern about how to get exact solution to the stress and strain of pipelines when it bears the internal and external pressure and axial force, the effects of high temperature and water hammer effect, failure forms etc. [10, 11]. Some researchers have obtained some excellent regularity on failure forms and damage mechanics through mathematical method and experimental method [12, 13]. But it is difficult to find the accurate solution by these analytical methods. The specifications of existing steel wire reinforced pipelines are unified, so it is also difficult to obtain the regularity of data by us- ing experiment methods. Therefore the finite element analysis method may be the better way to discuss the comprehensive performance of steel wire reinforced composite pipelines.

This paper presents a mathematic model of the composite pipe's mechanical properties based on basic equations of elastic mechanics and establishes 3D CAD models of two kinds of steel wire reinforced composite pipes, and analyzes the displacements and stress distribution of the pipes under constant internal and external pressure by using FEA software, respectively. The differences between the mechanical properties of the two kinds of composite pipes are elaborated by comparing the displacements of the distribution curve along their corresponding paths.

\section{Constitutive Models}

The cylindrical composite tube has been presented in the cylindrical coordinate system for analysis and modeling purposes, where the cylinder coordinates are: $\gamma$ the radial, $\theta$ the circumferential, and $z$ the axial coordinates of cylinder. When pipelines are subjected to axisymmetric loading, the stresses and strains are independent of $\theta$, and the radial and axial displacements depend only on the radial and axial coordinate, respectively. So the radial, hoop and axial displacement of the tube can be expressed as:

$$
\begin{aligned}
& u_{\gamma}=u_{\gamma}(\gamma) \\
& u_{\theta}=u_{\theta}(z, \gamma) \\
& u_{z}=u_{z}(z)
\end{aligned}
$$

Based on the theory of elastic mechanics, using the cylindrical coordinate system shown in Figure 2, the stress and strain transformation of the tube cell is given by

$\left[\begin{array}{c}\sigma_{z} \\ \sigma_{\theta} \\ \sigma_{\gamma} \\ \tau_{\theta \gamma} \\ \tau_{z \gamma} \\ \tau_{z \theta}\end{array}\right]=\left[\begin{array}{cccccc}E_{11} & E_{12} & E_{13} & 0 & 0 & E_{16} \\ E_{21} & E_{22} & E_{23} & 0 & 0 & E_{26} \\ E_{31} & E_{32} & E_{33} & 0 & 0 & E_{36} \\ 0 & 0 & 0 & E_{44} & E_{45} & 0 \\ 0 & 0 & 0 & E_{54} & E_{55} & 0 \\ E_{61} & E_{62} & E_{63} & 0 & 0 & E_{66}\end{array}\right]\left[\begin{array}{c}\varepsilon_{z} \\ \varepsilon_{\theta} \\ \varepsilon_{\gamma} \\ \gamma_{\theta \gamma} \\ \gamma_{z \gamma} \\ \gamma_{z \theta}\end{array}\right]$

The stress can be written as

$$
\left\{\begin{array}{l}
\sigma_{z}=E_{11} \varepsilon_{z}+E_{12} \varepsilon_{\theta}+E_{13} \varepsilon_{\gamma}+E_{16} \gamma_{z \theta} \\
\sigma_{\theta}=E_{21} \varepsilon_{z}+E_{22} \varepsilon_{\theta}+E_{23} \varepsilon_{\gamma}+E_{26} \gamma_{z \theta} \\
\sigma_{\gamma}=E_{31} \varepsilon_{z}+E_{32} \varepsilon_{\theta}+E_{33} \varepsilon_{\gamma}+E_{26} \gamma_{z \theta} \\
\tau_{\theta \gamma}=E_{44} \gamma_{\theta \gamma}+E_{45} \gamma_{z \gamma} \\
\tau_{z \gamma}=E_{54} \gamma_{\theta \gamma}+E_{55} \gamma_{z \gamma} \\
\tau_{z \theta}=E_{61} \varepsilon_{z}+E_{62} \varepsilon_{\theta}+E_{63} \varepsilon_{\gamma}+E_{66} \gamma_{z \theta}
\end{array}\right.
$$

For this model, the strain-displacements can be predi- 


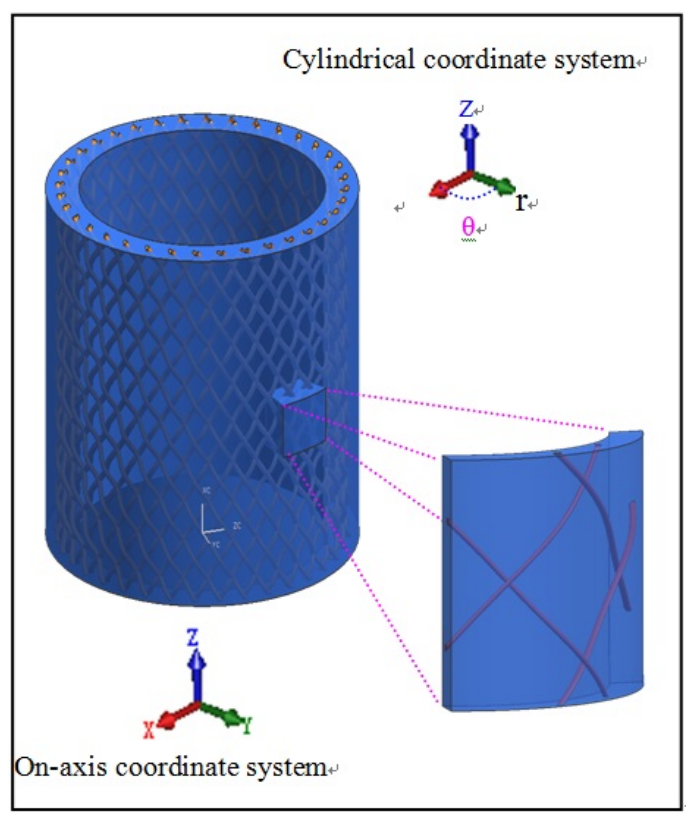

(a)

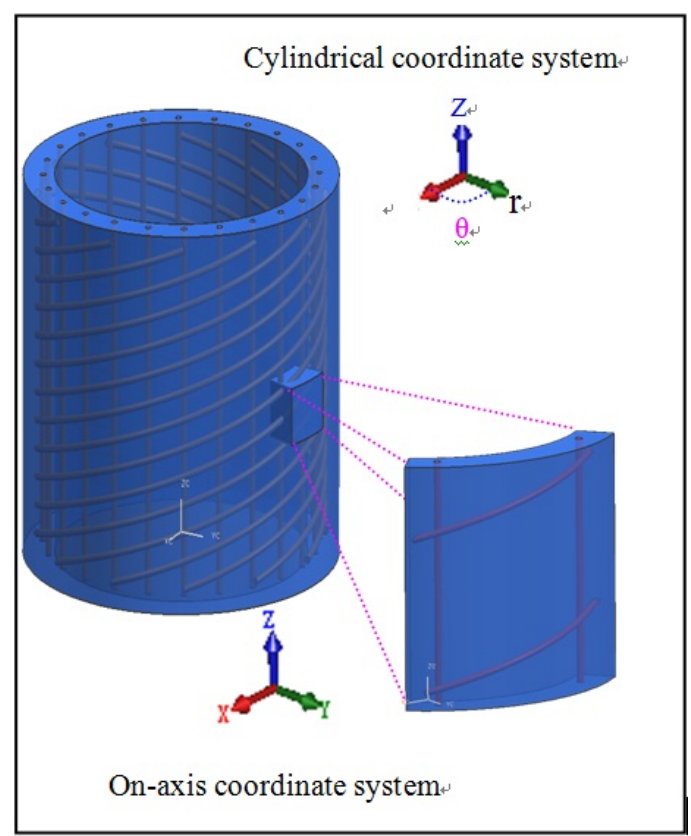

(b)

Figure 2 Model and coordinates: (a) Braided steel wire reinforced composite pipe, and (b) welded steel wire reinforced composite pipe gested as

$$
\left\{\begin{array}{l}
\varepsilon_{\gamma}=\frac{d u_{r}}{d r} \\
\varepsilon_{\theta}=\frac{u_{r}}{r} \\
\varepsilon_{z}=\frac{d u_{z}}{d z}=\varepsilon_{\theta} \\
\gamma_{z \gamma}=0 \\
\gamma_{\theta \gamma}=\frac{d u_{\theta}}{d r}-\frac{u_{\theta}}{r} \\
\gamma_{z \theta}=\frac{d u \theta}{d z}=r_{\theta} r
\end{array}\right.
$$

And the equilibrium equation for the current axisymmetric problem can be expressed as:

$$
\left\{\begin{array}{l}
\frac{d \sigma_{r}}{d r}+\frac{\sigma_{r}-\sigma_{\theta}}{r}=0 \\
\frac{d \tau_{\theta r}}{d r}+\frac{2 \tau_{\theta r}}{r}=0 \\
\frac{d \tau_{z r}}{d r}+\frac{\tau_{z r}}{r}=0
\end{array}\right.
$$

Substituting the expressions for the stress of Eq. (3) into Eq. (5), we can obtain the following differential equation

$$
\begin{aligned}
& u_{r}=A r^{\sqrt{\frac{E_{22}}{E_{33}}}}+B r^{-\sqrt{\frac{E_{22}}{E_{33}}}}+\frac{\frac{E_{12}-E_{13}}{E_{33}}}{1-\sqrt{\frac{E_{22}}{E_{33}}}} \varepsilon_{\theta} \\
&+\frac{\frac{E_{62}-E_{63}}{E_{33}}}{4-\frac{E_{22}}{E_{33}}} \gamma_{\theta} r^{2}
\end{aligned}
$$

where $A$ and $B$ are unknown constants of integration, and have to be determined from the boundary conditions.

Now the material modulus matrix elements $E_{i j}$ are needed, and the stress-strain relation with respect to the compliance matrix is

$$
\left[\begin{array}{c}
\varepsilon_{x} \\
\varepsilon_{y} \\
\varepsilon_{z} \\
\gamma_{x y} \\
\gamma_{x z} \\
\gamma_{y z}
\end{array}\right]=\left[\begin{array}{cccccc}
C_{11} & C_{12} & C_{13} & 0 & 0 & 0 \\
C_{21} & C_{22} & C_{23} & 0 & 0 & 0 \\
C_{31} & C_{32} & C_{33} & 0 & 0 & 0 \\
0 & 0 & 0 & C_{44} & 0 & 0 \\
0 & 0 & 0 & 0 & C_{55} & 0 \\
0 & 0 & 0 & 0 & 0 & C_{66}
\end{array}\right]\left[\begin{array}{c}
\sigma_{x} \\
\sigma_{y} \\
\sigma_{z} \\
\tau_{x y} \\
\tau_{x z} \\
\tau_{y z}
\end{array}\right]
$$

Suppose that the steel distributions are very similar in the $\mathrm{y}$ and $\mathrm{z}$ directions for the unidirectional orientation composite pipe. Then the matrix component values can be written as

$$
\begin{gathered}
C_{11}=1 / E_{x} \quad C_{22}=1 / E_{y} \quad C_{33}=1 / E_{z} \\
C_{12}=-\mu_{x y} / E_{x} \quad C_{13}=-\mu_{x z} / E_{x} \quad C_{23}=-\mu_{y z} / E_{y} \\
C_{44}=1 / G_{y z} \quad C_{55}=1 / G_{x z} \quad C_{66}=1 / G_{x y}
\end{gathered}
$$

And

$$
E_{z}=E_{y} \quad G_{x z}=G_{x y} \quad G_{y z}=\frac{E_{y}}{2\left(1+\mu_{y z}\right)}
$$

where $E_{x}, E_{y}, E_{z}$ are elastic modulus, $G_{i j}$ are shear modulus, and are Poisson's ratios.

$$
C_{12}=C_{13} \quad C_{22}=C_{33} \quad C_{55}=C_{66}
$$


Now we have

$$
E_{i j}=C_{i j}^{-1}
$$

The transformation of stiffness from on-axis coordinate system to cylindrical one can be obtained using a stiffness transformation matrix as

$$
\left[E_{r, z, \theta}\right]=\left[T_{\sigma}\right]^{-1}\left[E_{x, y, z}\right]\left[T_{\varepsilon}\right]
$$

where $T_{\sigma}$ and $T_{\varepsilon}$ are matrixes of the base change of stress and the strain, respectively, and are expressed as follows

$$
\begin{gathered}
{\left[T_{\sigma}\right]=\left[\begin{array}{cccccc}
m^{2} & n^{2} & 0 & 0 & 0 & 2 m n \\
n^{2} & m^{2} & 0 & 0 & 0 & -2 m n \\
0 & 0 & 1 & 0 & 0 & 1 \\
0 & 0 & 0 & m & -n & 1 \\
0 & 0 & 0 & n & m & 1 \\
-m n & m n & 0 & 0 & 0 & m^{2}-n^{2}
\end{array}\right]} \\
{\left[T_{\varepsilon}\right]=\left[\begin{array}{cccccc}
m^{2} & n^{2} & 0 & 0 & 0 & m n \\
n^{2} & m^{2} & 0 & 0 & 0 & -m n \\
0 & 0 & 1 & 0 & 0 & 1 \\
0 & 0 & 0 & m & -n & 1 \\
0 & 0 & 0 & n & m & 1 \\
-2 m n & 2 m n & 0 & 0 & 0 & m^{2}-n^{2}
\end{array}\right]}
\end{gathered}
$$

where $m=\cos \varphi, n=\sin \varphi$ and $\varphi$ is the cylindrical angle of the steel wire from the pipe axis. Here, we define the $\varphi$ of braided steel wire reinforced composite pipe is $45^{\circ}$, and $60^{\circ}$ of welded steel wire reinforced composite pipe.

So when the $A, B, E_{i j}, C_{i j}$ are obtained by determining the boundary conditions, the displacements, strains and stresses are thus obtained from Eqs. (6), (3), (4), respectively.

\section{Finite Element Analysis}

FEA Static analysis is performed to determine the displacements, stress, strains, and forces in structure or components caused by loads that do not induce significant inertia and damping effects. Steady load and response conditions are assumed in static analysis. The process of the static analysis is as follows:

(1) Modeling: The ANSYS is employed to perform FEA of the pipelines. In view of the fact that the steel wires embedded in the composite pipes are bodies of curved surface, which is difficult to build a perfect model even using parametric modeling method, so CAD models of the composite pipes are modeled in 3D CAD software, and then imported into ANSYS.

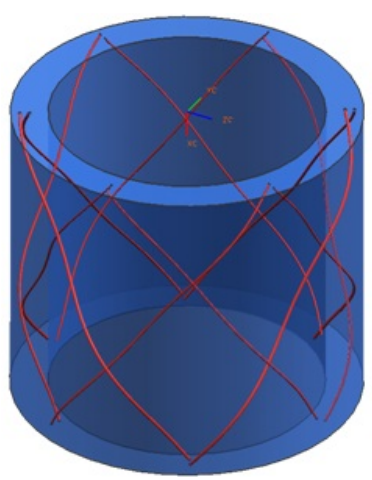

(a)

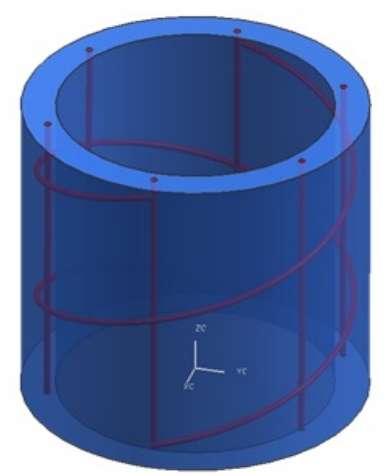

(b)
Figure 3 CAD Models: (a) Braided steel wire reinforced composite pipe, and (b) welded steel wire reinforced composite pipe

Table 1 Material Properties

\begin{tabular}{|c|c|c|}
\hline Property parameters & Plastic layer & Steel wire \\
\hline $\mathrm{E}(\mathrm{MPa})$ & 1002 & 210000 \\
\hline$\mu 2$ & 0.45 & 0.26 \\
\hline
\end{tabular}

The models are shown in Figure 3, where the diameter of steel wire is $1.5 \mathrm{~mm}$, inner diameter of composite pipe is $88 \mathrm{~mm}$, and outer diameter is $110 \mathrm{~mm}$, thus the thickness of the pipe is $11 \mathrm{~mm}$. The braided steel wire reinforced composite pipe is simplified as Figure 3(a), where there are 12 steel wires in the pipe, of which 6 are clockwise, and the others are counterclockwise. The helical angle of the steel wire is $45^{\circ}$. That is to say, they are perpendicular to each other in 3D space. The welded steel wire reinforced composite pipe is simplified as Figure 3(b), where there are 2 groups of steel wires within the pipe: one group contains 6 straight steel wires in longitudinal direction and a long cylindrical spiral steel wire in latitudinal direction, whose screw pitch is $100 \mathrm{~mm}$. The latitudinal steel wire intersects with the longitude ones. In order to simulate the welding effect, two kinds of pipes of $100 \mathrm{~mm}$ long are picked up as the sample.

(2) Materials selection: The composite pipelines contain two kinds of materials: steel and plastic, and they have different material properties. The materials property parameters are listed in Table 1, where E represents Young's modulus, and $\mu$ represents Poisson's ratio.

(3) Meshing: We choose hyperelastic 10-node-element No. 187 as plastic matrix elements, solid 10-nodeelement No. 92 as steel wire elements. The meshing results are shown in Figure. 4, where the braided steel wire reinforced composite pipe contains 129068 ele- 


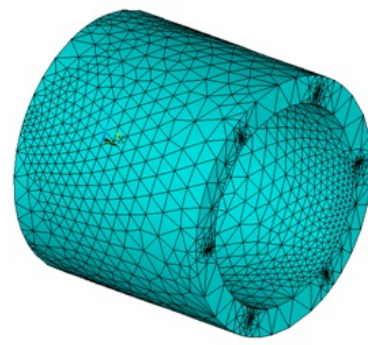

(a)

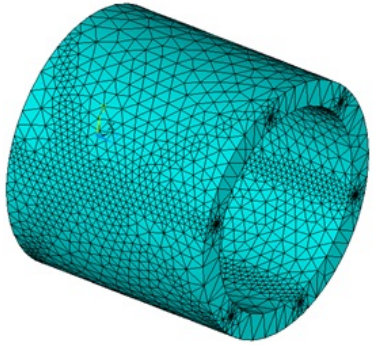

(b)

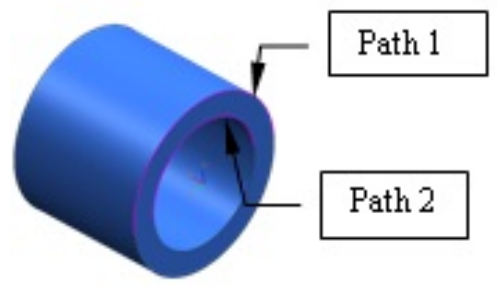

Figure 6 Paths
Figure 4 Finite Element models: (a) Braided steel wire reinforced composite pipe, and (b) welded steel wire reinforced composite pipe

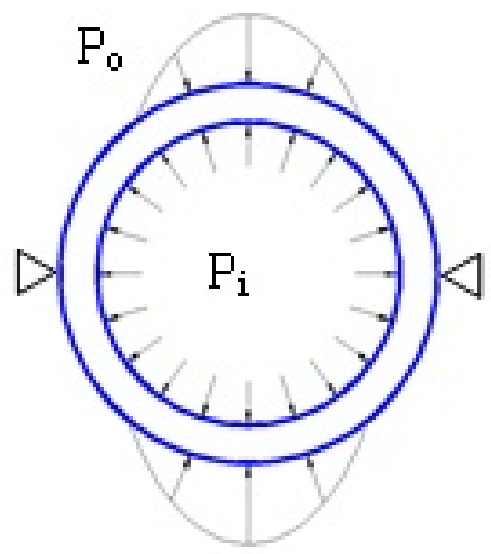

Figure 5 Schematic diagram of applying loads

ments and 178650 nodes; the welded steel wire reinforced composite pipe contains 326419 elements and 449531 nodes.

(4) Service conditions: As shown in Figure 5, the loads applied to the pipe are all structure loads. Firstly we need to restrict the DOF of the composite pipe by setting all the DOF of lines on both sides to zero. Then we apply the simplified actual pressure to the inside and outside surfaces. Pressures applied to the inside $(\mathrm{Pi})$ and outside surface $(\mathrm{Po})$ are $1 \mathrm{MPa}$ and 0 $0.8 \mathrm{MPa}$, respectively.

(5) Results Visualization: the displacement and stress of the composite pipe are analyzed by using ANSYS, and the results are shown in Table 2.

\section{Results and Discussion}

It's well known that under the same loadings, the smaller the displacement values, the better the mechanical properties of the composite pipe. In order to evaluate the reinforcing effects of the two kinds of composite pipelines, we need to compare the displace- ments at key positions, such as the boundary lines. Therefore we select the two paths on the boundary, as shown in Figure 6. The displacements of all nodes on path 1 and path 2 for the two kinds of composite pipes are extracted by using FEA software.

All the displacements in $X, Y$, and $Z$ directions, as well as resultant displacements of the two kinds of composite pipes under the same pressure are mapped onto path 1 and path 2, and then they can be plotted as shown in Figure 7, respectively. In Figure 7, $U X$ denotes the displacements in $x$-direction, $U Y$ denotes the displacements in $y$-direction, $U Z$ denotes the displacements in $z$-direction, and $U S U M$ denotes the resultant displacements.

The maximum displacements of the two kinds of composite pipes on Path 1 and Path 2 are listed in Table 3 .

From Table 3 and Figure 7, we can draw the following conclusions:

(1) The steel wires have the coincident displacement with the whole composite pipe. It is proven that in the model the steel wires are combined with plastic layers perfectly just like the actual composite pipe. The steel wires bear main pressure, so the mechanical properties of the composite pipe are determined by the quality of steel wire. The composite are destroyed when the steel wires are beyond bearing limitation.

(2) The stress of the braided steel wire reinforced composite pipe is more even than welded steel wire reinforced composite pipe. As the two group steel wires imbedded in the braided steel wire reinforced composite pipe are at the same helical angle, they are bearing the equal pressure. While for the welded steel wire reinforced composite pipe, the steel wire are longitudinal and latitudinal so the latitudinal steel wires bear more pressure than the longitudinal wires under radial pressure.

(3) The maximum displacements of the latter are larger than the former by about 2.3 times, and the changes of the displacements curves of both path 1 and path 2 are more regularly and more stable than 
Table 2 FEA Results

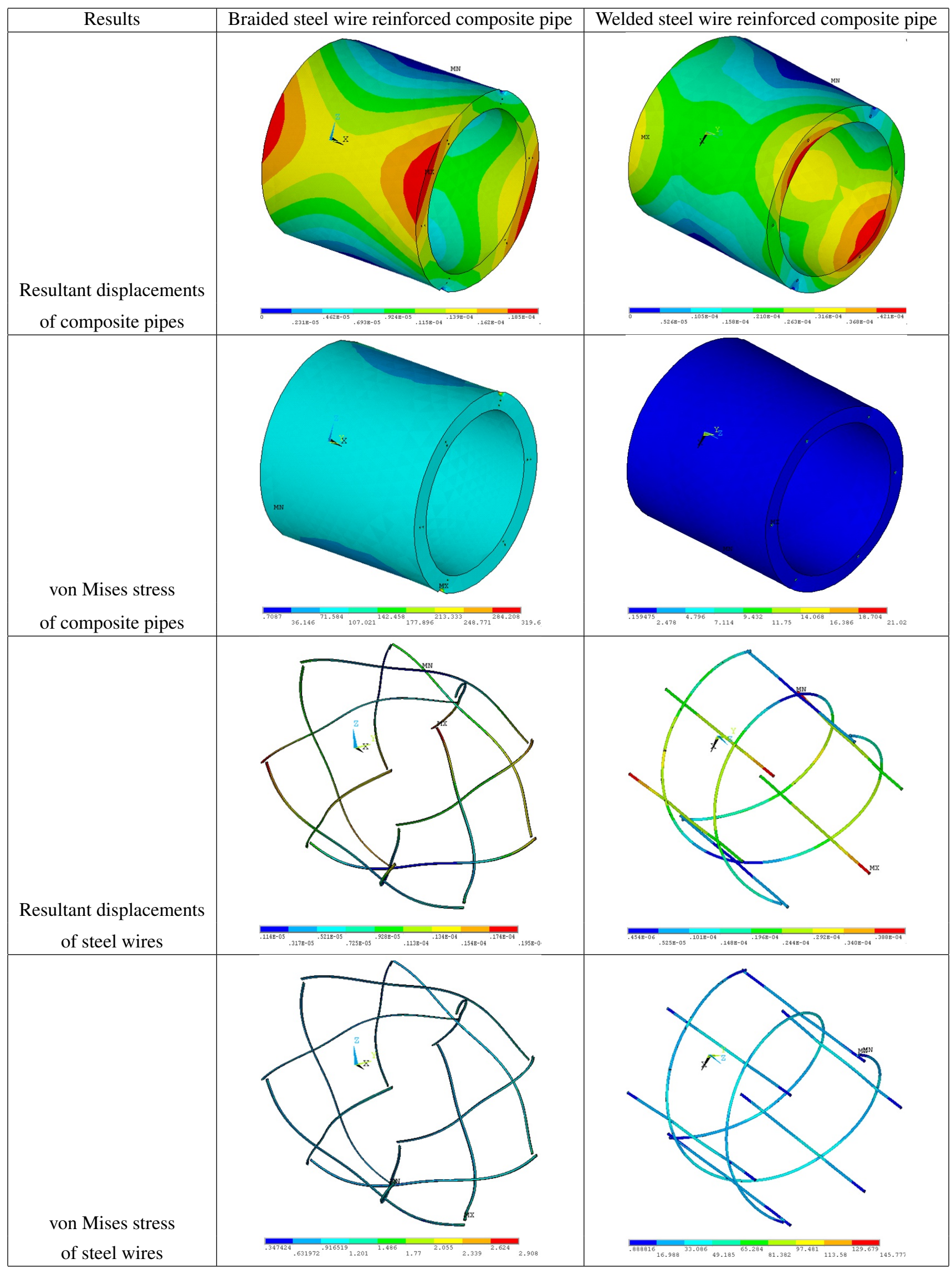




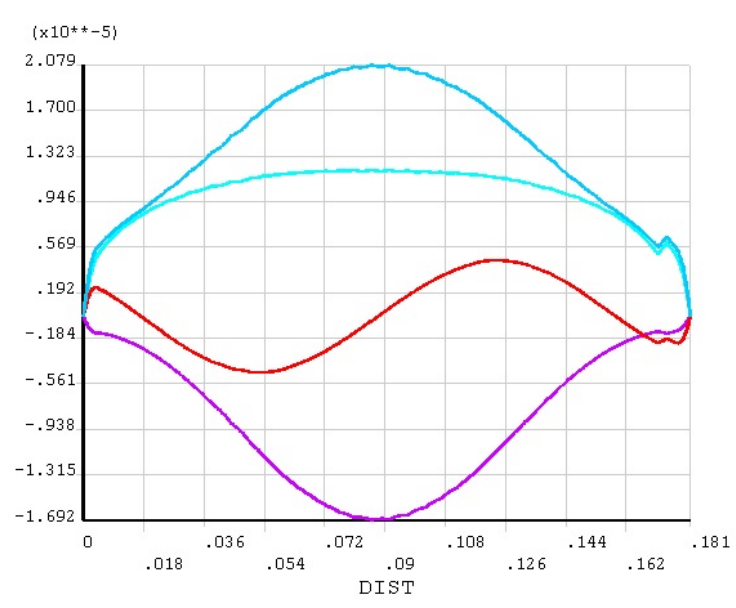

(a)

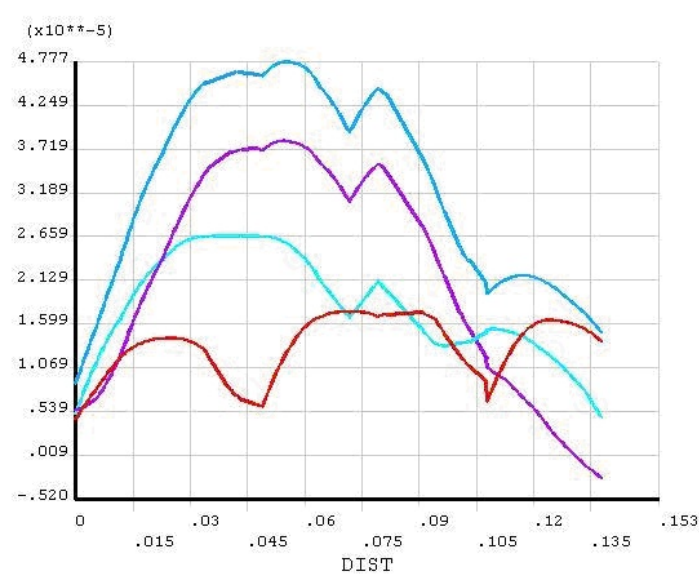

(c)

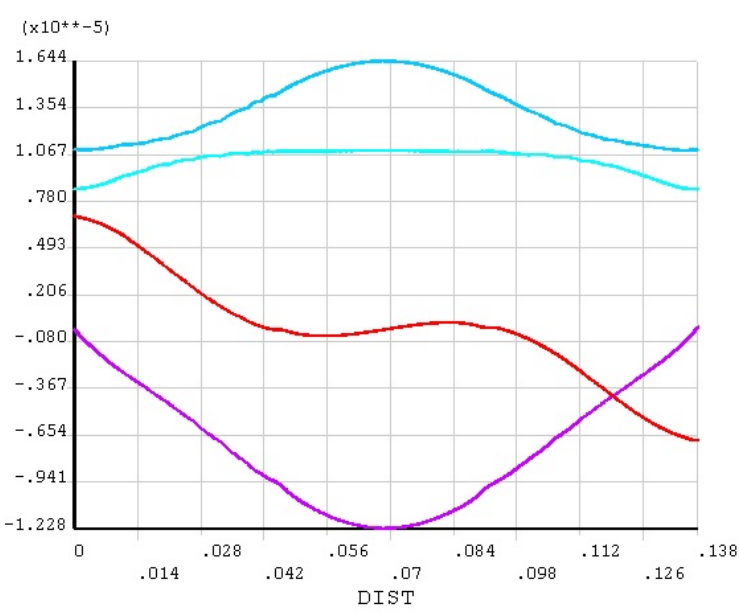

(b)

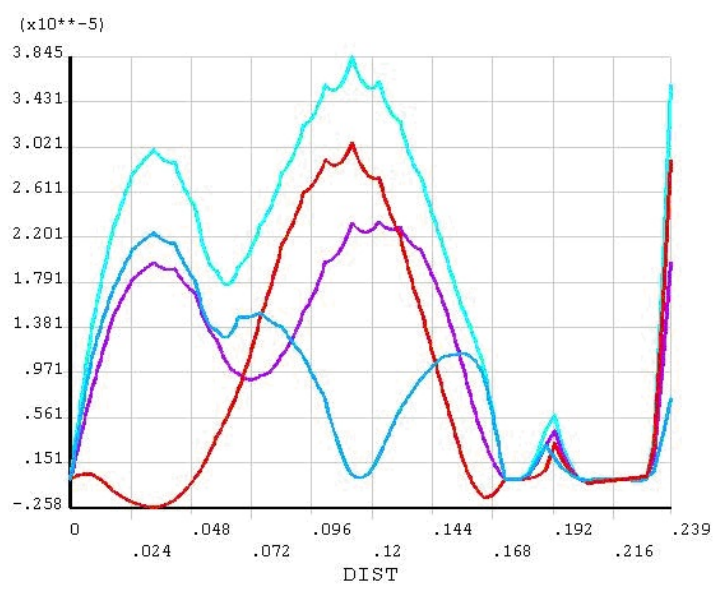

(d)

Figure 7 Displacements on paths: (a) Braided steel wire reinforced composite pipe's displacements on path 1, (b) Braided steel wire reinforced composite pipe's displacements on path 2, (c) welded steel wire reinforced composite pipe's displacements on path 1, and (d) welded steel wire reinforced composite pipe's displacements on path 2

Table 3 The maximum displacements of paths

\begin{tabular}{|c|c|c|}
\hline \multirow{2}{*}{} & \multicolumn{2}{|c|}{ Displacements (m) } \\
\cline { 2 - 3 } & $\begin{array}{c}\text { Braided steel wire } \\
\text { reinforced composite } \\
\text { pipe }\end{array}$ & $\begin{array}{c}\text { Welded steel wire } \\
\text { reinforced composite } \\
\text { pipe }\end{array}$ \\
\hline Path 1 & $2.079 \mathrm{E}-5$ & $4.777 \mathrm{E}-5$ \\
\hline Path 2 & $1.644 \mathrm{E}-5$ & $3.845 \mathrm{E}-5$ \\
\hline
\end{tabular}

the welded steel wire reinforced composite pipe. The former has better performances than the latter if applying the same pressure to both kinds of composite pipes.

\section{Conclusions}

This paper presents an mathematic model of the composite pipe's mechanical properties based on basic equa- tions of elastic mechanics, which can be used to solve the problems of stress, strain and displacements; Then the 3D CAD models of two kinds of steel wire reinforced composite pipes are established, and the displacements and stress distribution of the pipes under constant internal and external pressure are analyzed by using FEA software, respectively. The differences between the mechanical properties of the two kinds of composite pipes are elaborated by comparing the displacements of the distribution curve along their corresponding paths. The results show that the braided steel wire reinforced composite pipes have better mechanical properties than the welded ones. That may be due to the crisscross that makes both the clockwise and counter-clockwise steel wires bear the same pressure.

The future work will be focused on investigating 
the effect of the geometrical and structural parameters on the mechanical performance of the two composite pipes.

\section{References}

[1] Xia M, Takayanagi H, Kemmochi K. "Bending Behavior of Filament Wound Fiber Reinforced Sandwich Pipes". Compost Structures. 56 (2):201-210, 2002.

[2] H. Bakaiyan, H. Hosseini, E. Ameri. "Analysis of multi-layered filament-wound composite pipes under combined internal pressure and thermomechanical loading with thermal variation". Composite Structures. 88 (1): 532-541, 2009.

[3] Li Zhixiong. "Multi-layer winding plating steel reinforced plastic composite pipe". Beijing: intellectual property press, 2003.

[4] Gan Guogong. "Spiral wound steel wire reinforced plastic composite pipe". Beijing: intellectual property press, 2002.

[5] Huang Ze. "Buried type continuous winding steel wire reinforced plastic pipe". Beijing: intellectual property press, 2003.
[6] Lin Xiufeng, "The stress analysis and Optimization for plastic-steel composite pipe: [Master thesis]". Zhejiang University, 2006.

[7] Zheng J, Lu Y, Li X. "Experiment research for mechanical properties of Wire spiral reinforced plastic pipe". China Plastic, 20 (6): 82-88, 2006.

[8] HG/T369022001. Steel framework reinforced PE pipe for industry.

[9] SY/T666222006. Steel framework reinforced PE pipe for oil and gas transportation.

[10] Wei S, Han Q, Wei X. "Researches on composite pipe. Materials", 17 (9): 64-67, 2003.

[11] Wei R, Wang X, Hang B. "The problems of steel frame reinforced composite pipe". Building Material of Chemical, 12 (2): 54-56, 1996.

[12] Li Houbu, Yan Milin, Qi Dongtao. "Failure analysis of steel wire reinforced thermoplastics composite pipe”. Engineering Failure Analysis. 20(1): 88-96, 2012.

[13] George Z. Voyiadjis, Peter I. Kattan. "Mechanics of small damage in fiber-reinforced composite materials". Composite Structures. 92(1): 2187-2193, 2010. 